\title{
AUDET, Louis-Philippe, Histoire du Conseil de l'Instruction publique. Éditions Leméac, Montréal, 1964.346 p. Avant-propos, bibliographie, table des matières (IX-XIX), appendices, conclusion, postface, index analytique.
}

\section{Lionel Groulx et J. Rémillard}

Volume 18, numéro 1, juin 1964

URI : https://id.erudit.org/iderudit/302354ar

DOI : https://doi.org/10.7202/302354ar

Aller au sommaire du numéro

Éditeur(s)

Institut d'histoire de l'Amérique française

ISSN

0035-2357 (imprimé)

1492-1383 (numérique)

Découvrir la revue

Citer ce compte rendu

Groulx, L. \& Rémillard, J. (1964). Compte rendu de [AUDET, Louis-Philippe, Histoire du Conseil de l'Instruction publique. Éditions Leméac, Montréal, 1964. 346 p. Avant-propos, bibliographie, table des matières (IX-XIX), appendices, conclusion, postface, index analytique.] Revue d'histoire de l'Amérique française, 18(1), 155-160. https://doi.org/10.7202/302354ar d'utilisation que vous pouvez consulter en ligne. 
AUDET, Louis-Philippe, Histoire du Conseil de l'Instruction publique. Avant-propos, bibliographie, table des matières (IX-XIX), appendices, conclusion, postface, index analytique. Editions Leméac (Montréal, 1964), 346 p.

Faut-il le dire ? L'on ne s'attendait point à un procès presque en règle du Conseil de l'Instruction publique, à un plaidoyer pour l'école étatisée, à une apologie-préface au nouveau régime d'enseignement qui s'instaure dans l'Etat du Québec. Pourquoi, dans un ouvrage d'ailleurs très méritant, $M$. Audet nous révèle-t-il, dès le début, une intention trop évidente ? L'objectivité de l'historien n'en devient point pour tout cela forcément diminuée; elle souffre de cet apriorisme. L'ouvrage est fortement documenté, si même il ne l'est trop, donnant à penser qu'il s'y trouve peut-être plus de documentation que d'histoire. Non content de la centaine de pages de ses Appendices, l'auteur a voulu citer, en cours de route, nombre de documents qu'il eut suffi de résumer, ou de présenter l'essentiel. Un lecteur quelque peu impatienté aurait grande envie de citer à ce propos un passage de Henri-I. Marrou, dans De la Connaissance historique:

... notre histoire scientifique ne serait pas tombée si bas dans l'estime générale... si les travailleurs 
sérieux n'avaient pas à ce point méprisé leur public, ne s'étaient pas si souvent contentés de verser sur lui, sous le nom de livres, de simples tombereaux de fiches, rudis indigestaque moles. Trop de publications ne sont pas de l'histoire, mais seulement un ensemble de matériaux à demi-dégrossis avec quoi l'élaborer.

Indiquerai-je une première et grave lacune en cette histoire d'un régime scolaire ? On regrette, à chaque période, l'absence de statistiques sur le progrès de l'enseignement. Il y eut, croyonsnous et malgré tout, quelques progrès, ces progrès fussent-ils lents même trop lents, en deçà de légitimes espérances. A ne lire que les lacunes, hélas trop réelles des systèmes tentés depuis près d'un siècle et demi, on pourrait croire à une sorte d'immobilisme de l'enseignement dans le Bas-Canada ou le Québec. Chaque époque, même l'époque contemporaine, a pourtant marqué une notable avance. Au lendemain de 1841 et même auparavant et longtemps après, le mal suprême restera le manque de maîtres et l'absence d'écoles où les former, et ce, dans l'enseignement primaire et secondaire. La première école normale se verra balayée par la tourmente de 1837. Il faudra attendre 21 ans pour voir réapparaître les écoles normales primaires; l'école normale d'enseignement secondaire date sa fondation de 1940 ou 1941, croyons-nous. Sur ce point, l'auteur a-t-il rendu pleinement justice à Mgr Bourget qui, dès 1842, en vue de pourvoir à cette pénurie de maîtres, fait appel aux congrégations de France et aide à la fondation de 4 ou 5 d'origine canadienne-française ? Encore qu'à l'heure où nous sommes, le Québec se sente mal préparé à ses tâches actuelles: manque d'hommes tout court, manque de grands fonctionnaires, manque de techniciens, manque d'ingénieurs, manque de chefs d'entreprise, le régime du Conseil de l'Instruction publique en doit-il seul porter la responsabilité ? Il y a déjà plus d'un demi-siècle que l'état s'est chargé, lui-même, de ce que l'on a appelé "l'enseignement spécialisé". Si cet enseignement n'a pas répondu à l'attente d'aujourd'hui, à qui la faute ? Au reste, comment préparer les "spécialistes" qui, en réalité, nous manquent si affreusement, quand nos gouvernants n'osaient entreprendre sérieusement la reprise de nos ressources naturelles et que les "spécialistes" sortis des grandes écoles n'apercevaient guère de carrières où s'employer, si ce n'est en acceptant la manne du capital étranger ? Et les budgets de l'enseignement, et en ceci ni le Conseil de l'Instruction publique ni les évêques n'avaient pas seuls à y voir, ont-ils toujours été ce qu'ils auraient dû être? Dès 1843 le Rapport Sicotte dénonce 
l'insuffisance du budget de l'éducation. Et il ose rappeler aux gouvernants que "l'Etat doit considérer l'instruction des masses comme le premier besoin de la société" (22). Ce pitoyable état de chose s'est-il tellement amélioré au cours des années ? Celui qui écrit ces lignes n'a pas oublié l'image squelettique d'une université découverte un jour sur le Mont-Royal, déjà entourée d'une forêt de jeunes arbres, ses fenêtres crevées. Elle était là depuis dix ans, restée inachevée. Les catholiques montréalais, à la suite d'un double appel de leurs évêques, avaient pourtant fourni au moins dix millions pour la construction de leur université. L'Etat avait refusé de verser le reste, sous prétexte que l'édifice était mal situé et d'une envergure estimée trop vaste. Et, pour le dire en passant, l'on s'est vivement plaint, en ces derniers temps, de la multiplicité et voire de la confusion des autorités en notre système scolaire. Encore cette fois, à qui la faute ? La loi de 1875 avait reconnu le droit exclusif du Conseil de l'Instruction publique de légiférer en matière d'enseignement. Cependant, c'est fait reconnu: le Parlement continue de légiférer et de plus belle. A ce point qu'une double législation, ai-je noté dans mon Enseignement français au Canada, tome 1er, pages 294-295, va même s'édifier: l'une qui recherche encore le placet du Conseil de l'Instruction publique; l'autre, non la moindre, qui se garde bien du circuit traditionnel par les Comités. On fera de même pour le budget que le Conseil est censé préparer. Dès 1895 le gouvernement institue un nouveau fonds scolaire et prétend s'en réserver la distribution. Ces dérogations à une loi statutaire et par l'autorité gouvernementale elle-même, si louables qu'elles paraissent à M. Audet, ne pouvaient que semer l'incohérence dans la législation scolaire et gêner l'initiative de la surintendance. On ira plus loin: divers ministères, le secrétariat de la province, la trésorerie, le ministère des travaux publics, celui des terres et forêts, celui de la jeunesse se mettront à fonder des écoles de la catégorie des écoles spéciales, écoles qui relèveront des divers ministères qui les auront fondées. Qu'en un pareil régime il y ait eu chevauchement, confusion des autorités, en quoi le Conseil de l'Instruction publique en peut-il être tenu responsable ? Quant aux critiques de M. Audet sur les organismes issus de la loi de 1875 , nous ne sommes pas éloignés de lui donner raison. Peut-être eût-il mieux valu, selon le conseil de Mgr Bourget, que l'épiscopat de la province se tînt en dehors du Conseil, quitte à se servir de son influence et de celle de son clergé, comme le voulait l'Evêque de Montréal, pour surveiller et vivifier de l'extérieur l'enseignement public. Il pouvait être sage de maintenir, coûte que coûte, l'enseignement en dehors de 
la politique, hors du patronage sordide et de la lutte des partis et des changements de gouvernement. Il était peut-être moins opportun de confier la haute régie à des hommes laïcs et chefs d'Eglise déjà trop pris par leur devoir d'état, pour s'occuper activement de problèmes aussi complexes, aussi vivants, aussi sujets aux évolutions que l'instruction publique. Le vice était là plus que dans les excellentes intentions des législateurs de 1875. A ce propos, l'on eût bien fait d'accepter et dès le commencement, le conseil d'Urgèle Archambault, fondateur de l'Ecole polytechnique, qui eût souhaité compléter le grand organisme par un "Conseil de perfectionnement composé exclusivement d'hommes d'écoles".

Vais-je relever, et la chose en vaut-elle la peine, cette autre critique de M. Audet qui estime étrange, voire anticonstitutionnel, qu'un corps non élu par le peuple se soit vu confier la distribution des subventions scolaires? L'Auteur oublie d'observer que cette distribution ne s'accomplit qu'indirectement de sommes dûment votées par la Législature. Et où se trouve la différence entre cette distribution effectuée par un corps indépendant et la distribution par le député ou l'organisateur électoral, et ce, au nom du patronage politicien, ainsi que la mode ne tardera pas à s'établir ? Au surplus, et il serait bon de s'en souvenir, la mystique démocratique n'avait pas alors subjugué les esprits comme en nos temps. Elle n'était pas devenue un mythe sacré. Et l'on ne croyait pas qu'il suffît à une institution d'appartenir à l'essence démocratique pour qu'on lui conférât toutes les vertus.

Il conviendrait aussi, quelle que soit la passion étatisante, de moins laisser croire que les évêques, partisans d'un organisme indépendant de l'Etat dans le domaine de l'enseignement, se portaient en définitive et par-dessus tout, à la défense d'un fief. Ils ne sont pas les seuls, du reste, à prendre cette position; ils sont d'accord, M. Audet le reconnaît, avec une large portion, sinon avec la majorité de l'opinion et d'un grand nombre d'hommes politiques. Il serait facile d'établir que l'épiscopat redoutait de bonne foi l'intrusion de la politique dans un champ où elle est peu chez soi. Félix-Gabriel Marchand, promoteur d'un projet de ministère de l'instruction publique en 1897 , ne se fait pas faute de reconnaître les bonnes intentions des évêques. "Certes, avoue-t-il, à Mgr Merry del Val, nous nous faisons un devoir de le reconnaître hautement, nos évêques ont à cœur le bien général du peuple, et personne plus qu'eux n'a de zèle pour le développement de notre pays..." Si les évêques, ainsi que l'affirme M. Audet, éprouvent une "sainte peur" de la politique du temps, 
dès que celle-ci s'approche du domaine de l'éducation, c'est qu'à tort ou à raison, un certain radicalisme n'a pas laissé de les effrayer. Et l'on ne saurait reprocher à des évêques de croire engagée, dans ce débat autour de l'enseignement, l'âme de leur peuple. Que quelques-uns d'entre eux n'aient point pratiqué l'intransigeance outre mesure à l'égard de l'Etat, l'exemple de $\mathrm{Mgr}$ Bourget en témoigne qui n'eût point voulu les évêques dans le Conseil de l'Instruction publique. Et il y a aussi cet aveu de l'évêque de Chicoutimi, Mgr Jean Langevin, le prélat le plus écouté alors en la matière, que "dans ce pays, à raison de la nature même de la population, il est impossible d'assurer pratiquement à l'Eglise ses droits dans toute leur plénitude" (87) .

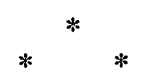

M. Audet a vu, dans l'histoire de l'enseignement au Québec, une évolution laborieuse. Tout fut, dans l'histoire du Québec, il eût pu le noter, évolution laborieuse. Misères d'un peuple qui, après 1760 , eut à se réadapter en tous les compartiments de sa vie. Laborieuse son adaptation à ses divers régimes politiques qui lui furent imposés de haut: quête incessante d'une liberté qui ne cherchait qu'à lui échapper. Laborieuse, l'adaptation de son régime juridique ou judiciaire aux conditions nouvelles, sociales et autres qui lui étaient faites, effort sans cesse repris pour sauver la loi, le droit qui était le sien, partie intégrante de ses traditions vitales. Laborieuse l'adaptation de son Eglise, de sa liberté religieuse, en un milieu qui ne lui était pas toujours des plus favorables. Nullement étonnante, après tout cela, la laborieuse adaptation de son régime scolaire dans le milieu géographique, social, culturel où la conquête anglaise l'avait placé. Que les essais, les tâtonnements ne se soient point multipliés, le contraire serait miracle. Le miracle, ce serait bien plutôt qu'un peuple d'une conscience nationale fatalement troublée, anémiée, ait pu quand même persévérer dans la ligne de sa culture. M. Audet salue avec espoir le nouveau régime scolaire qui prend position. Il y voit tous les éléments d'une réforme dynamique, opportunément appropriée aux exigences d'un Etat moderne. Personne n'entend bouder cet espoir.

Nous avons peut-être fait de cette Histoire $d u$ Conseil de l'Instruction publique, une critique sévère, sinon même trop sévère. L'ouvrage ne manque pas d'observations très justes. Et reconnaissons qu'en dépit de ses lacunes et peut-être même de son esprit, cette histoire, ne serait-ce que par sa surabondante docu- 
mentation, rendra grand service aux étudiants. M. Audet est un laborieux. Il vient de le prouver une fois de plus. Qu'il se débarrasse de l'usage souvent outré qu'il se permet de l'intégration du document dans le texte de ses ouvrages. Et il lui manquera peu de chose pour se classer parmi nos meilleurs historiens.

LIONEL GROULX, ptre 